\title{
Acknowledgment to reviewers
}

\section{Lee Spector ${ }^{1}$}

Published online: 7 March 2019

○ Springer Science+Business Media, LLC, part of Springer Nature 2019

Genetic Programming and Evolvable Machines relies on generous contributions of time and expertise by scholars who agree to review articles on a voluntary basis. Over the past year the following people have made such contributions:

Lee Altenberg

Ignacio Arnaldo

Filipe Assunção

R. Muhammad Atif Azad

Benjamin Bach

Juhee Bae

Bendeguz Bak

Wolfgang Banzhaf

Marcio Barros

Michael Behrisch

Peter Bentley

Heder Soares Bernardino

Lonni Besançon

H.-G. Beyer

Anastasia Bezerianos

Leonardo Bezerra

Anthony Brabazon

Ivona Brajevic

J. Branke

Radim Burget

Stefano Cagnoni

Alberto Cano

Mauro Castelli

Edward Clarkson

Manuel Clergue
German Creamer

Grant Dick

John Drake

Marc Ebner

Anna Esparcia-Alcazar

Francisco Fernández de Vega

Cyril Fonlupt

Stephanie Forrest

James Foster

Mohammad Ghoniem

Pascal Goffin

Erik Goodman

Ian Graham

Steven Gustafson

Zulfiqar Habib

Pauline Haddow

I. Harvey

Thomas Helmuth

Malcolm Heywood

J. Ignacio Hidalgo

Christian Jacob

Domagoj Jakobović

Dong Hyun Jeong

Colin Johnson
Tatiana Kalganova

Ahmed Kattan

D. Keymeulen

Mark Kotanchek

Krzysztof Krawiec

Gabriel Kronberger

William La Cava

Raúl Lara-Cabrera

Andre Leier

Antonios Liapis

Fernando Lobo

Michael Lones

Nuno Lourenço

Sean Luke

Evelyne Lutton

Penousal Machado

Luca Manzoni

Virginie Marion-Poty

James McDermott

Nicholas McPhee

Eric Medvet

Nathalie Mejean

Luana Micallef

Julian Miller

David Montana

Lee Spector

1spector@hampshire.edu

1 School of Cognitive Science, Hampshire College, Amherst, MA 01002, USA 


$\begin{array}{lll}\text { Jason Moore } & \text { Ruben Salvador } & \text { Alberto Tonda } \\ \text { Voitech Mrazek } & \text { Frederic Saubion } & \text { Leonardo Trujillo } \\ \text { Xuan Hoai Nguyen } & \text { Marc Schoenauer } & \text { Paulo Urbano } \\ \text { Mohammed Omari } & \text { Eric Schulte } & \text { Tommaso Urli } \\ \text { Michael O’Neill } & \text { Kisung Seo } & \text { Andrew Vande Moere } \\ \text { Fernando Otero } & \text { Kevin Sim } & \text { Leonardo Vanneschi } \\ \text { Matheus Paixao } & \text { Moshe Sipper } & \text { Yuan Wang } \\ \text { Gisele Pappa } & \text { Aidan Slingsby } & \text { P. Whigham } \\ \text { David Alejandro Pelta } & \text { T. Soule } & \text { David White } \\ \text { Nelishia Pillay } & \text { Giovanni Squillero } & \text { John Woodward } \\ \text { Craig Reynolds } & \text { Thomas Stutzle } & \text { Bing Xue } \\ \text { Denis Robilliard } & \text { Jerry Swan } & \text { Shin Yoo } \\ \text { Peter Rockett } & \text { Ivan Tanev } & \text { Landaez Ysmaldo Jose } \\ \text { Katya Rodríguez Vázquez } & \text { Necmi Taşpinar } & \text { Tina Yu } \\ \text { C. Ryan } & \text { Daniel Tauritz } & \text { Mengjie Zhang }\end{array}$

I thank these reviewers for their service to the Genetic Programming and Evolvable Machines journal and to the research community.

Lee Spector

Editor-in-Chief, Genetic Programming and Evolvable Machines

Publisher's Note Springer Nature remains neutral with regard to jurisdictional claims in published maps and institutional affiliations. 